\title{
Manifolds with Bakry-Emery Ricci Curvature Bounded Below
}

\author{
Issa Allassane Kaboye1, Bazanfaré Mahaman² \\ ${ }^{1}$ Faculté de Sciences et Techniques, Université de Zinder, Zinder, Niger \\ ${ }^{2}$ Département de Mathématiques et Informatique, Université Abdou Moumouni, Niamey, Niger \\ Email: allassanekaboye@yahoo.fr,bmahaman@yahoo.fr
}

How to cite this paper: Kaboye, I.A. and Mahaman, B. (2016) Manifolds with Bakry-Emery Ricci Curvature Bounded Below. Advances in Pure Mathematics, 6, 754-764. http://dx.doi.org/10.4236/apm.2016.611061

Received: August 24, 2016

Accepted: October 14, 2016

Published: October 17, 2016

Copyright $\odot 2016$ by authors and Scientific Research Publishing Inc. This work is licensed under the Creative Commons Attribution International License (CC BY 4.0).

http://creativecommons.org/licenses/by/4.0/

\begin{abstract}
In this paper we show that, under some conditions, if $M$ is a manifold with BakryÉmery Ricci curvature bounded below and with bounded potential function then $M$ is compact. We also establish a volume comparison theorem for manifolds with nonnegative Bakry-Émery Ricci curvature which allows us to prove a topolological rigidity theorem for such manifolds.
\end{abstract}

\section{Keywords}

Bakry Émery Ricci Curvature, Myers Theorem, Volume Comparison Theorem, Topological Rigidity Theorem

\section{Introduction}

Let $(M, g)$ be a complete Riemannian manifold and $f: M \rightarrow R$ a smooth function. A Bakry-Émery Ricci curvature is defined by $R i c_{f}=R i c_{g}+$ Hessf , where $R i c_{g}$ stands the Ricci curvature of $(M, g)$ and Hessf denotes the Hessian of $f$. The function $f$ is called the potential function. For simplicity, denote Ric $_{g}$ by Ric.

The Bakry-Émery tensor occurs in many different subjects, such as diffusion processes and Ricci flow.

When $f$ is a constant function, the Bakry-Émery Ricci tensor becomes the Ricci tensor so it is natural to investigate which geometric and topological results for the Ricci tensor extend to the Bakry-Émery Ricci tensor.

As an extension of Ricci curvature, many classical results in Riemannian geometry asserted in terms of Ricci curvature have been extended to the analogous ones on Bakry-Émery Ricci curvature condition.

In [1] G. Wei and W. Wylie proved some comparison theorems for smooth metric 
measure spaces with Bakry-Émery Ricci tensor bounded below. In this paper we establish a Myers type theorem for manifolds bounded below by a negative constant. Therefore we prove that is a generalization of the theorem of M. Limoncu in [2] or H. Tadano in [3].

In the second part of this paper we establish a condition on noncompact manifold with nonnegative Bakry-Émery Ricci curvature to be diffeomorphic to the euclidean space $\mathbb{R}^{n}$.

\section{Mains Results}

The following theorem is a similar theorem proved in [4] and [5] and is a generalization of Myers theorem.

Theorem 2.1. Let $\left(M, g, \mathrm{e}^{-f} d v o l_{g}\right)$ be a metric space such that Ric R $_{f} \geq-(n-1) k^{2}$. Suppose that $M$ contains a ball $B\left(x_{0}, r\right)$ of center $x_{0}$ and radius $r$ such that the mean curvature $m(r)$ of the geodesic sphere $S\left(x_{0}, r\right)$ with respect the inward pointing normal vector verifies $m(r)<-(n-1) k$.

If there exists a constant $c \geq 0$ such that $|f| \leq c$ then $M$ is compact and

$$
\operatorname{diam}(M) \leq 2 r+\frac{\ln \left\lfloor\left(h_{0}-k\right) /\left(h_{0}+k\right)\right\rfloor}{2 k}
$$

where $h_{0}=\sup _{x \in S(p, r)} \frac{m(x)}{n-1}$.

It is well known that there exist noncompact manifolds with nonnegative Ricci curvature which are not finite topological type. Recall that a manifold $M$ is said to have finite topological type if there is a compact domain $\Omega$ whose boundary $\partial \Omega$ is a topological manifold such that $M \backslash \Omega$ is homeomorphic to $\partial \Omega \times[0,+\infty)$. An important result about topological finiteness of a complete Riemannian manifold $M$ is due to Abresch and Gromoll (See [6]).

Let $f$ be a potential function on $M$ satisfying $|f(x)| \leq c \cdot d(p, x)$ for some nonnegative constant $c$ and a fixed point $p$.

$$
\text { Set } h(x)=f(x)+3 \cdot d(p, x) \text {; let } \alpha_{f}^{p}=\lim _{r \rightarrow \infty} \frac{\operatorname{Vol}_{h}(B(p, r))}{\omega_{n} r^{n}} \text { and } \alpha_{f}(M)=\inf _{p} \alpha_{f}^{p} \text {. }
$$

In this paper we show a topological rigidity theorem for noncompact manifolds with nonnegative Bakry-Émery Ricci curvature as follow:

Theorem 2.2. Let $\left(M, g, \mathrm{e}^{-f} d v o l_{g}\right)$ be a metric space such that $\operatorname{Ric}_{f}(x) \geq 0$. Suppose $\alpha_{M}>0$ and $K_{p} \geq-k^{2}$ for a point $p \in M$ and $|f(x)| \leq c d(p, x)$. If for all $r>0$

$$
\frac{\operatorname{Vol}_{h}(B(p, r))}{\omega_{n} r^{n}}<\left\{1+2^{-n}\left(\frac{1}{8 k r} \ln \left(\frac{2}{1+\mathrm{e}^{-2 k r}}\right)\right)^{n-1}\right\} \alpha_{f}(M)
$$

then $M$ is diffeomorphic to $\mathbb{R}^{n}$.

\section{Proofs}

Proof of theorem 2.1. The techniques used in the proof of this theorem are based on 
[4] and [5]. First, let construct a comparison model space. Let $S^{m-1}$ be the unit sphere in $\mathbb{R}^{m}$ and take a real $r$ and $R \in \mathbb{R} \cup\{+\infty\}$ so that $0<r<R \leq \infty$. Let $\phi$ be the solution of the differential equation

$$
\phi^{\prime \prime}(t)-k^{2} \phi(t)=0
$$

with initial values $\phi(r)=a_{0}$ and $\phi^{\prime}(r)=a_{1}<0$. Suppose $\phi(t) \geq 0$ for all $t \in[r, R)$. Hence

$$
\phi(t)=\frac{a_{0}}{2 k}\left[\left(\frac{a_{1}}{a_{0}}+k\right) \mathrm{e}^{k(t-r)}+\left(k-\frac{a_{1}}{a_{0}}\right) \mathrm{e}^{-k(t-r)}\right] .
$$

On $S^{m-1} \times[r, R)$ we define a Riemannian metric tensor by

$$
g_{(u, t)}=\phi(t) g_{c a n}^{m-1} \otimes t^{2}
$$

where $g_{c a n}^{m-1}$ is the standard metric on $S^{m-1}$.

Thus the Riemannian incomplete manifold $S^{m-1} \times[r, R)$ is with Ricci curvature constant equal to $-(m-1) k^{2}$.

For all $s \geq r$, the hypersurface $S^{m-1} \times\{s\}$ of $S^{m-1} \times[r, R)$ with mean curvature vector with outward pointing vector i.e. with pointing positive $s$

$$
\bar{H}(u, s)=-(m-1) \frac{\phi^{\prime}(s)}{\phi(s)} .
$$

Now let prove, under the hypotheses of theorem 2.1, that $M$ is compact.

Let $y$ be an arbitrary point in $M \backslash S(p, r)$; there exists a point $x \in S(p, r)$ such that $d(y, S(p, r))=d(x, y)$. Let $\gamma_{1}$ be a minimal geodesic joining $x$ to $y$; $\gamma_{1}(t)=\exp _{x}(t-r) u$ with $u \in T_{x} S(p, r)^{\perp}$ and $\|u\|=1$.

Let $\left(\dot{\gamma}_{1}(r), e_{1}, \cdots, e_{n-1}\right)$ be a parallel orthonormal frame along $\gamma_{1}$ and set

$Y_{i}(t)=\left.\frac{\mathrm{d}}{\mathrm{d} s} \exp _{x} t e_{i}(s)\right|_{s=0}$. Hence $Y_{i}$ is a $S(p, r)$-Jacobi field along $\gamma_{1}$. The geodesic $\gamma_{1}$ can be extend to a minimal geodesic $\gamma$ starting at $p: \gamma(t)=\exp _{p} t v$ with $u=T_{x} \operatorname{exprv}$ (see [4], Proposition 3) and $Y_{i}$ is a $S(p, r)$-Jacobi field along $\gamma_{1}$ if and only if $Y_{i}$ can be extended to a Jacobi field along $\gamma$, null at $p$.

In the geodesic polar coordinates the volume element can be written as:

$$
\mathrm{d} v o l_{g}=A(t, \theta) \mathrm{d} t \wedge \mathrm{d} \theta^{n-1}
$$

where $\mathrm{d} \theta^{n-1}$ is the volume form on the unit sphere $S^{n-1}$ and

$$
\begin{aligned}
& A(t, \theta)=\left\|Y_{1}(t) \wedge \cdots \wedge Y_{n-1}(t)\right\| \text {. Hence } \\
& \mathrm{e}^{-f} \mathrm{~d} v o l g=A_{f}(t, \theta) \mathrm{d} t \wedge \mathrm{d} \theta^{n-1}=\mathrm{e}^{-f} A(t, \theta) \mathrm{d} t \wedge \mathrm{d} \theta^{n-1} \text {. We have } \\
& \frac{\mathrm{d}}{\mathrm{d} t} \ln (A(t, \theta))=\frac{A^{\prime}(t, \theta)}{A(t, \theta)}=\sum_{i=1}^{n-1}\left\langle Y_{i}^{\prime}(t), Y_{i}(t)\right\rangle=m(t) \\
& \frac{\mathrm{d}}{\mathrm{d} t} \ln \left(A_{f}(t, \theta)\right)=\frac{A_{f}^{\prime}(t, \theta)}{A_{f}(t, \theta)}=m_{f}(t)
\end{aligned}
$$

To prove the theorem 2.1 we use the following theorem proved by G. Wei and W. 
Wylie in [1].

Theorem 3.1. (Mean Curvature Comparison). Let p be a point in M. Assume

$$
\operatorname{Ric}_{f}(\partial r, \partial r) \geq(n-1) H \text {. }
$$

1) If $\partial_{r} f \geq-a(a \geq 0)$ along a minimal geodesic segment from $\mathrm{p}$ (when $H>0$ assume $r \leq \frac{\pi}{2 \sqrt{H}}$ ) then

$$
m_{f}(r) \leq m_{H}(r)+a
$$

along that minimal geodesic segment from $\mathrm{p}$. Equality holds if and only if the radial sectional curvatures are equal to $\mathrm{H}$ and $f(t)=f(p)-a t$ for all $t<r$.

2) If $|f| \leq c$ along a minimal geodesic segment from $\mathrm{p}$ and $H<0$ or $H>0$ and $r \leq \frac{\pi}{4 \sqrt{H}}$ then

$$
m_{f}(r) \leq m_{H}^{n+4 c}(r)
$$

along that minimal geodesic segment from $p$.

3) If $|f| \leq c$ along a minimal geodesic segment from $\mathrm{p}$ and $H>0$ and $r \in\left[\frac{\pi}{4 \sqrt{H}}, \frac{\pi}{2 \sqrt{H}}\right]$ then

$$
m_{f}(r) \leq\left(1+\frac{4 c}{n-1}\right) \cdot m_{H}(r)+4 c \sqrt{H}
$$

In particular when $H=0$ we have

$$
m_{f}(r) \leq \frac{n+4 c-1}{r}
$$

where $m_{H}^{n+4 c}$ is the mean curvature of the geodesic sphere in $M^{n+4 c}$ the simply connected model space of dimension $n+4 c$ with constant curvature $H$ and $m_{H}$ is the mean curvature of the model space of dimension $n$.

In fact in [1] G. Wei and W. Wylie stated that, if $r \in\left[\frac{\pi}{4 \sqrt{H}}, \frac{\pi}{2 \sqrt{H}}\right]$ then

$$
\int_{0}^{r} f(t)\left(s n_{H}^{2}\right)^{\prime \prime}(t) \mathrm{d} t \leq c\left(\frac{2}{\sqrt{H}}-s n_{H}(2 r)\right)
$$

where $s n_{H}(t)$ is the solution of equation $y^{\prime \prime}(t)+H y(t)=0$.

From theorem 3.1 above and Equations ((8) and (9)) for all $s \geq r$, we have:

$$
\frac{A_{f}(s, \theta)}{A_{f}(r, \theta)}=\mathrm{e}^{\int_{r}^{s} m_{f}(t, \theta) \mathrm{d} t} \leq \mathrm{e}^{\int_{r}^{s} m_{-k^{2}}^{n+4 c}(t, \theta) \mathrm{d} t}=\frac{A_{-k^{2}}^{n+4 c}(s, \theta)}{A_{-k^{2}}^{n+4 c}(r, \theta)} .
$$

where $A_{-k^{2}}^{n+4 c}(s, \theta)$ denotes the volume element in the space of dimension $n+4 c$ and constant Ricci curvature $-(n-1) k^{2}$. From the assumption we have: $A_{-k^{2}}^{n+4 c}(s, \theta)=(\phi(s))^{n+4 c-1}$.

If $h=\frac{\phi^{\prime}(r)}{\phi(r)}<-k$ then $\phi(s) \rightarrow 0$ when $s \rightarrow R=r+\frac{1}{2 k} \ln \left[\frac{\left(h_{0}-k\right)}{\left(h_{0}+k\right)}\right]$. 
Hence there exists $R_{0} \leq R$ so that $A\left(R_{0}, \theta\right)=0$ which means that there exists $i_{0}$ so that the $S(p, r)$-Jacobi field $Y_{i_{0}}$ vanishes at $\gamma\left(R_{0}\right)$. Therefore we conclude that $\gamma\left(R_{0}\right)$ is a conjugate point of the center $p$ of the sphere $S(p, r)$. Hence $\gamma$ ceases to be minimal, that is $d(p, y) \leq R=r+\frac{1}{2 k} \ln \left[\frac{\left(h_{0}-k\right)}{\left(h_{0}+k\right)}\right]$ and $\operatorname{Diam}(M) \leq 2 r+\frac{1}{k} \ln \left[\frac{\left(h_{0}-k\right)}{\left(h_{0}+k\right)}\right]$.

In [2] M. Limoncu generalized a classical Myers theorem by using the Bakry-Émery Ricci curvature tensor on complete and connected Riemannian manifolds $(M, g)$. This theorem can be viewed as a corollary of theorem 2.1.

Corollary 3.2. Let $(M, g)$ be a complete and connected Riemannian manifold of dimension n. If there exists a smooth function $f: M \rightarrow \mathbb{R}$ satisfying the inequalities

$$
\text { Ric }+\operatorname{Hess}(f) \geq(n-1) k^{2}>0
$$

and $|f| \leq c$ then $M$ is compact.

Proof of Corollary

To prove this corollary it suffices to show that there exist a positive real $\epsilon$ with $\epsilon<k$ and a geodesic sphere $S(p, r)$ which mean curvature verifies $m_{f}(r)<-(n-1) \epsilon$.

Let $x$ be a point in $M$ and let $\gamma:[0, r] \rightarrow M$ be a minimal geodesic joining $p$ to $x$ and $\left(e_{i}(t)\right)_{1 \leq i \leq n-1}$ be a parallel orthonormal vector fields along $\gamma$ orthonormal to $\gamma$.

Set $Y_{i}(t)=\phi(t) e_{i}(t)$ where $\phi(t)=\sin \left(\frac{\pi}{2 r} t\right)$. We have

$$
\begin{aligned}
\Delta r(x) & \leq \sum_{i=1}^{n-1} I\left(Y_{i}, Y_{i}\right) \leq \int_{0}^{r}(n-1) \phi^{\prime 2}(t)-\phi^{2}(t) \operatorname{Ric}\left(\gamma^{\prime}(t)\right) \\
& \leq \int_{0}^{r}(n-1)\left(\phi^{\prime}(t)^{2}-k^{2} \phi^{2}(t)\right)+\int_{0}^{r} \phi^{2}(t) \operatorname{Hess}(f) \mathrm{d} t \\
& =(n-1) \int_{0}^{r}\left(\frac{\pi^{2}}{4 r^{2}} \cos ^{2} \frac{\pi}{2 r} t-k^{2} \sin ^{2} \frac{\pi}{2 r} t\right) \mathrm{d} t+\phi^{2}(r) \partial_{r} f-2 \int_{0}^{r} \phi^{\prime}(t) \phi(t) \partial_{t} f \mathrm{~d} t \\
& \leq(n-1) \int_{0}^{r}\left(\frac{\pi^{2}}{4 r^{2}} \cos ^{2}\left(\frac{\pi}{2 r} t\right)-k^{2} \sin ^{2} \frac{\pi}{2 r} t\right) \mathrm{d} t+\partial_{r} f+2 c \int_{0}^{r}\left|d\left(\phi^{\prime}(t) \phi(t)\right)\right| \mathrm{d} t \\
& \leq(n-1) \frac{\pi^{2}}{8 r}-(n-1) \frac{k^{2} r}{2}+\partial_{r} f+c \frac{\pi^{2}}{2 r^{2}} \int_{0}^{r / 2} \cos \left(\frac{2 \pi}{r} t\right)-c \frac{\pi^{2}}{2 r^{2}} \int_{r / 2}^{r} \cos \left(\frac{2 \pi}{r} t\right) \\
& \leq \frac{n-1}{8 r} \pi^{2}-\frac{(n-1) k^{2}}{2} r+\partial_{r} f+\frac{c \pi}{r} .
\end{aligned}
$$

Therefore

$$
m_{f}(r)=\Delta r-\partial_{r} f \leq \frac{n-1}{8 r} \pi^{2}-\frac{(n-1) k^{2}}{2} r+\frac{c \pi}{r}
$$

which allows that $m_{f}(r)<0$ if $r>\frac{\pi}{2 k} \sqrt{1+\frac{8 c}{(n-1) \pi}}$.

By Compactness of $S(p, r)$, there exists a positive constant $\epsilon^{\prime}$ so that, for any 
geodesic $\gamma$ emanating from $p$ we have $m_{f}(r) \leq-(n-1) \epsilon^{\prime}<0$

Since $\operatorname{Ric}_{f} \geq(n-1) k^{2}>-(n-1) \epsilon^{\prime 2}$, the conclusion follows from theorem 2.1.

Corollary 3.3. (E. Calabi)

Let $(M, g)$ be a complete and connected Riemannian manifold of dimension $\mathrm{n}$. Suppose there exists a smooth function $f: M \rightarrow \mathbb{R}$ so that $|f| \leq c$ and $\operatorname{Ric}_{f} \geq 0$. If $M$ is noncompact then there exists a geodesic $\gamma$ in $M$ so that $\liminf t^{2} \operatorname{Ric}_{f}\left(\gamma^{\prime}(t)\right) \leq n-1$.

Proof

It is clear that, if for a geodesic $\gamma$ issuing from $p$ there exist two positive reals $k$ and $r$ so that $\operatorname{Ric}_{f}\left(\gamma^{\prime}(t)\right) \geq(n-1) k^{2}$ for all $t \geq r$ then $p$ admits a conjugate point along $\gamma$. Hence, if $M$ is noncompact, for all $p \in M$, there exists a geodesic $\gamma$ issuing from $p$ so that for any two positive real $k$ and $r$ there exists $t \geq r$ so that $\operatorname{Ric}_{f}\left(\gamma^{\prime}(t)\right)<(n-1) k^{2}$. In particular if $k=\frac{1}{t}=\frac{1}{r}$ we take $\operatorname{Ric}_{f}\left(\gamma^{\prime}(t)\right)<\frac{n-1}{t^{2}}$ and the conclusion follows.

\section{Corollary 3.4. (Ambrose)}

Let $(M, g)$ be a complete and connected Riemannian manifold of dimension $\mathrm{n}$. Suppose there exists a function $f$ on $M$ so that Ric $_{f} \geq 0$. If there exists a point $p$ in $M$ so that, for any geodesic $\gamma$ emanating from $p$, parametrized by it's arc-length we have

$$
\int_{0}^{+\infty} \operatorname{Ric}_{f}\left(\gamma^{\prime}(t)\right) \mathrm{d} t=+\infty
$$

then $M$ is compact.

Proof

If $M$ is noncompact, from corollary 3.3, there exists $r_{0}>0$ so that $\operatorname{Ric}_{f}\left(\gamma^{\prime}(t)\right)<\frac{n-1}{t^{2}}$ for $t \geq r_{0}$. Therefore,

$$
\int_{0}^{+\infty} \operatorname{Ric}_{f}\left(\gamma^{\prime}(t)\right) \mathrm{d} t \leq \int_{0}^{r_{0}} \operatorname{Ric}_{f}\left(\gamma^{\prime}(t)\right) \mathrm{d} t+\int_{r_{0}}^{+\infty} \frac{n-1}{t^{2}} \mathrm{~d} t<+\infty .
$$

\section{Proof of theorem 2.2}

Let $\operatorname{Vol}_{f}(B(p, s))=\int_{B(p, s)} \mathrm{e}^{-f} \mathrm{~d} v o l_{g}$ denotes the weighted volume of the geodesic ball of center $p$ and radius $s$ in $M$ and $\operatorname{vol}_{H}^{m}(s)$ the volume of geodesic ball of radius $s$ in the model space $M_{H}^{m}$ with constant curvature $H$ and dimension $m$.

In Differential Geometry, the volume comparison theory plays an important rule. Many important results in this topic can not be obtained without volume comparison results as topological rigidity results.

For complete smooth metric measure space with Ric $_{f} \geq 0$ the following lemma improved the volume comparison theorem proved by G. Wei and W. Wylie In [1]:

Lemma 3.5. Let $\left(M, g, \mathrm{e}^{-f} d v o l g\right)$ be complete smooth metric measure space with Ric $_{f} \geq 0$. Fix $p \in M$; if there exists $c$ so that $|f(x)| \leq c d(p, x)$ then for $R \geq r>0$

$$
\frac{\operatorname{Vol}_{f}(B(p, R))}{\operatorname{Vol}_{f}(B(p, r))} \leq \mathrm{e}^{3 c}\left(\frac{R}{r}\right)^{n} .
$$

Proof 
Let $x$ be a point in $M$ and let $\gamma:[0, r] \rightarrow M$ be a minimal geodesic joining $p$ to $x$ and $\left(e_{i}(t)\right)_{1 \leq i \leq n-1}$ be a parallel orthonormal vector fields along $\gamma$ orthonormal to $\gamma$. Set $Y_{i}(t)=\frac{t}{r} e_{i}(t)$.

By the second variation formula we have:

$$
\begin{aligned}
m(r) & =\Delta r \leq \sum_{i=1}^{n-1} I\left(X_{i}, X_{i}\right) \\
& =\int_{0}^{r} \sum_{i=1}^{n-1}\left\|X_{i}^{\prime}(t)\right\|-\left\langle R\left(X_{i}(t), \gamma^{\prime}(t)\right) \gamma^{\prime}(t), X_{i}(t)\right\rangle \mathrm{d} t \\
& \leq \frac{1}{r^{2}} \int_{0}^{r}\left(n-1-t^{2} R i c\left(\gamma^{\prime}\right)\right) \mathrm{d} t \\
& \leq \frac{n-1}{r}+\int_{0}^{r} \frac{t^{2}}{r^{2}} \operatorname{Hess}(f)\left(\gamma^{\prime}, \gamma^{\prime}\right) \mathrm{d} t \\
& \leq \frac{n-1}{r}+\int_{0}^{r} \frac{t^{2}}{r^{2}}(f \circ \gamma)^{\prime \prime} \mathrm{d} t \\
& =\frac{n-1}{r}+\frac{1}{r^{2}} \int_{0}^{r} \frac{\mathrm{d}}{\mathrm{d} t}\left(t^{2}(f \circ \gamma)^{\prime}\right)(t) \mathrm{d} t-\frac{2}{r^{2}} \int_{0}^{r} t(f \circ \gamma)^{\prime}(t) \mathrm{d} t \\
& =\frac{n-1}{r}+\partial_{r} f-\frac{2}{r} f(x)+\frac{2}{r^{2}} \int_{0}^{r}(f \circ \gamma) \mathrm{d} t \\
& \leq \frac{n-1}{r}+\partial_{r} f+3 c .
\end{aligned}
$$

Hence $m_{f}(r)=\Delta r-\partial_{r} f \leq \frac{n-1}{r}+3 c$. From (9) and the above relation, we have $m_{f}(t)=\frac{\mathrm{d}}{\mathrm{d} t} \ln \left(A_{f}(t, \theta)\right)=\frac{A_{f}^{\prime}(t, \theta)}{A_{f}(t, \theta)} \leq \frac{n-1}{t}+3 c$.

For all positive reals $r$ and $s$, integrating this relation we have:

$$
\int_{r}^{s} m_{f}(t) \mathrm{d} t=\frac{A_{f}(s, \theta)}{A_{f}(r, \theta)} \leq \mathrm{e}^{3 c}\left(\frac{s}{r}\right)^{n-1} .
$$

Therefore we have $r^{n-1} A_{f}(s, \theta) \leq \mathrm{e}^{3 c} A_{f}(r, \theta) s^{n-1}$. Hence

$$
\int_{0}^{R} \int_{S^{n-1}} r^{n-1} A_{f}(s, \theta) \mathrm{d} \theta \mathrm{d} r \leq \mathrm{e}^{3 c} \int_{0}^{R} \int_{S^{n-1}} A_{f}(r, \theta) s^{n-1} \mathrm{~d} \theta \mathrm{d} r
$$

which implies

$$
\frac{R^{n}}{n} \int_{s^{n-1}} A_{f}(s, \theta) \mathrm{d} \theta \leq \mathrm{e}^{3 c} s^{n-1} \int_{0}^{R} \int_{s^{n-1}} A_{f}(r, \theta) \mathrm{d} \theta \mathrm{d} r=\mathrm{e}^{3 c} s^{n-1} v o l_{f}(B(p, R)) .
$$

and integrating from 0 to $R^{\prime}$ with respect to $s$ we obtain the conclusion.

Set $h(x)=f(x)+3 c \cdot d(p, x)$. Then

$$
m_{h}(r)=\Delta_{h} r=\Delta r-\partial_{r} h=\Delta_{f} \cdot r-3 c \leq \frac{n-1}{r} .
$$

Hence we have

$$
\frac{\operatorname{Vol}_{h}(B(p, R))}{\operatorname{Vol}_{h}(B(p, r))} \leq\left(\frac{R}{r}\right)^{n}
$$


From the relation (28) we deduce that the function $r \rightarrow \frac{v_{h}(B(p, r))}{\omega_{n} r^{n}}$ is nonincreasing.

Let $\alpha_{f}^{p}=\lim _{r \rightarrow \infty} \frac{\operatorname{Vol}_{h}(B(p, r))}{\omega_{n} r^{n}} \leq \frac{\operatorname{Vol}_{h}(B(p, 1))}{\omega_{n}}$ and $\alpha_{f}(M)=\inf _{p} \alpha_{f}^{p}$.

We have $0 \leq \alpha_{f}(M) \leq \frac{\operatorname{Vol}_{h}(B(p, 1))}{\omega_{n}}$.

We say that $M$ is of large weighted volume growth if $\alpha_{f}(M)>0$.

Let $R_{p, t}$ be the set of the unit initial tangent vectors to the geodesics starting from $\mathrm{p}$ which are minimized at least to $t$ and $R_{p, t}^{c}$ its complementary set. Set

$$
B_{R_{p, r}}(p, r)=\left\{x \in B(p, r), \exists \gamma:[0, s] \rightarrow M \quad \text { minimal, } \gamma(0)=p, \gamma^{\prime}(0) \in U_{p}\right\} .
$$

Let $\Sigma_{p}$ a subset of the unit sphere $U_{p} \subset T_{p} M$. Set

$$
B_{\Sigma_{p}}(p, r)=\left\{x \in B(p, r), \exists \gamma:[0, s] \rightarrow M \quad \text { minimal, } \gamma(0)=p, \gamma^{\prime}(0) \in \Sigma_{p}\right\} .
$$

Lemma 3.6. If $|f(x)| \leq c \cdot d(p, x)$ and Ric $_{f} \geq 0$ then

1) the function $r \rightarrow \frac{\operatorname{Vol}_{h}\left(B_{\Sigma_{p}}(p, r)\right)}{\omega_{n} r^{n}}$ is nonincreasing and

2) for any $r>0, \frac{\operatorname{vol}_{h}\left(B_{R_{p, r}}(p, r)\right)}{\omega_{n} r^{n}} \geq \alpha_{f}^{p}$ where $h$ is defined by:

$h(x)=f(x)+3 c \cdot d(p, x)$.

Proof

By Equation (27) we have

$$
\frac{\mathrm{d}}{\mathrm{d} t} \ln A_{h}(t, \theta)=m_{h}(t) \leq \frac{n-1}{t}=\frac{\mathrm{d}}{\mathrm{d} t} \ln t^{n-1}
$$

hence we deduce that the function $t \rightarrow \frac{A_{h}(t, \theta)}{t^{n-1}}$ is decreasing.

By lemma 3 in [7] we have:

$$
\frac{\int_{\Sigma_{p}} \int_{0}^{R} A_{h}(t, \theta) \mathrm{d} t \mathrm{~d} \theta}{\int_{\Sigma_{p}} \int_{0}^{r} A_{h}(t, \theta) \mathrm{d} t \mathrm{~d} \theta} \leq \frac{\int_{\Sigma_{p}} \int_{0}^{R} t^{n-1} \mathrm{~d} t \mathrm{~d} \theta}{\int_{\Sigma_{p}} \int_{0}^{r} t^{n-1} \mathrm{~d} t \mathrm{~d} \theta}=\left(\frac{R}{r}\right)^{n}=\frac{\omega_{n} R^{n}}{\omega_{n} r^{n}} .
$$

Therefore

$$
\frac{\operatorname{Vol}_{h}\left(B_{\Sigma_{p}}(p, R)\right)}{\operatorname{Vol}_{h}\left(B_{\Sigma_{p}}(p, r)\right)}=\frac{\int_{\Sigma_{p}} \int_{0}^{\min [R, c u t \theta]} A_{h}(t, \theta) \mathrm{d} t \mathrm{~d} \theta}{\int_{\Sigma_{p}} \int_{0}^{\min [r, c u t \theta]} A_{h}(t, \theta) \mathrm{d} t \mathrm{~d} \theta} \leq \frac{\int_{\Sigma_{p}} \int_{0}^{R} A_{h}(t, \theta) \mathrm{d} t \mathrm{~d} \theta}{\int_{\Sigma_{p}} \int_{0}^{r} A_{h}(t, \theta) \mathrm{d} t \mathrm{~d} \theta} .
$$

For $0<r_{1} \leq r_{2}$ we have $\Sigma_{p}\left(r_{2}\right) \subset \Sigma_{p}\left(r_{1}\right)$ and by part (1) of the lemma 3.6 we have:

$$
\frac{\operatorname{vol}_{h} B_{\Sigma_{p}\left(r_{2}\right)}\left(p, r_{2}\right)}{\operatorname{vol}_{h} B\left(p, r_{2}\right)} \leq \frac{\operatorname{vol}_{h} B_{\Sigma_{p}\left(r_{2}\right)}\left(p, r_{1}\right)}{\operatorname{vol}_{h} B\left(p, r_{1}\right)} \leq \frac{\operatorname{vol}_{h} B_{\Sigma_{p}\left(r_{1}\right)}\left(p, r_{1}\right)}{\operatorname{vol}_{h} B\left(p, r_{1}\right)}
$$

and the part (2) can be proved as the lemma 3.10 in [8]. 
Lemma 3.7. Let $(M, g)$ be a complete noncompacte Riemannian manifold and $f$ a potential function on $M$ with $|f(x)| \leq c d(p, x)$ and Ric $\geq 0$. If $M$ is of large weighted volume then

$$
\frac{\operatorname{Vol}_{h}\left(B_{\Sigma_{p}[\infty)}(p, r)\right)}{\omega_{n} r^{n}} \geq \alpha_{f}(M), \quad \forall r>0 .
$$

Proof

We have

$$
\operatorname{Vol}_{h}\left(B_{\Sigma_{p}(r)}(p, r)\right)=\operatorname{Vol}_{h}\left(B_{\Sigma_{p}(r)}(p, r) \backslash B_{\Sigma_{p}(\infty)}(p, r)\right)+\operatorname{Vol}_{h}\left(B_{\Sigma_{p}(\infty)}(p, r)\right)
$$

and

$$
\begin{aligned}
& V_{h} B_{\Sigma_{p}(r) \backslash \Sigma_{p}(\infty)}(p, r)=\int_{\Sigma_{p}(r) \backslash R_{p}} \int_{0}^{r} A_{h}(t, \theta) \mathrm{d} t \mathrm{~d} \theta \\
& \leq \int_{\Sigma_{p}(r) \backslash R_{p}} \int_{0}^{r} t^{n-1} \mathrm{~d} t \mathrm{~d} \theta \leq \frac{r^{n}}{n} \operatorname{Vol}\left(\Sigma_{p}(r) \backslash \Sigma_{p}(\infty)\right) .
\end{aligned}
$$

Since $\Sigma_{p}(\infty)=\bigcap_{r>0} \Sigma_{p}(r)$ we have $\lim _{r \rightarrow \infty} \operatorname{Vol}_{h}\left(\Sigma_{p}(r) \backslash \Sigma_{p}(\infty)\right)=0$; hence

$$
\alpha_{f}(M) \leq \lim _{r \rightarrow \infty} \frac{\operatorname{Vol}_{h}\left(B_{\Sigma_{p}(r)}(p, r)\right)}{\omega_{n} r^{n}}=\lim _{r \rightarrow \infty} \frac{\operatorname{Vol}_{h}\left(B_{\Sigma_{p}(\infty)}(p, r)\right)}{\omega_{n} r^{n}} .
$$

Lemma 3.8. Let $(M, g)$ be a complete noncompacte Riemannian manifold and $f$ a potential function on $M$ with $|f(x)| \leq c d(p, x)$ and Ric $_{f} \geq 0$. If $M$ is of large weighted volume then for any $x \in \partial B(p, r)$ we have

$$
d\left(x, \mathcal{R}_{p}\right) \leq 2 \alpha_{f}(M)^{-\frac{1}{n}}\left\{\frac{\operatorname{Vol}_{h} B(p, r)}{\omega_{n} r^{n}}-\alpha_{f}(M)\right\}^{1 / n} r .
$$

The proof of this lemma is step by step similar to the one in [9] (lemma 2.4).

Let $p, q$ be two points in $M$. The excess function is defined as:

$$
e_{p q}(x)=d(p, x)+d(q, x)-d(p, q) .
$$

By triangle inequality the excess function is nonnegative and is lipschitz. Let $\gamma$ be a ray from $p$ and set $s(x)=d(x, \gamma)$. Hence, for any $t \geq 0$ we have:

$$
e_{p, \gamma(t)}(x)=d(p, x)+d(\gamma(t), x)-t .
$$

The function $e_{p, \gamma(t)}$ is nonincreasing on $t$ and $e_{p, \gamma(t)}(x) \geq 0$.

Set $e_{p, \gamma}(x)=\lim _{t} e_{p, \gamma(t)}(x)$.

By the fact that $e_{p, \gamma(t)}$ is nonincreasing on $t$, we have $e_{p, \gamma}(x) \leq e_{p, \gamma(t)}(x), \forall t>0$.

Applying the Toponogov's theorem and the definition of critical point we have:

Lemma 3.9. Let $M$ be a complete noncompacte Riemannian manifold such that $K_{p}^{\min } \geq-k^{2}$ for some $k \neq 0$ and $p \in M$. Suppose that $x \neq p$ is a critical point of $d_{p}$. Then for any ray $\gamma$ issuing from $p$, we have

$$
e_{p \gamma}(x) \geq \frac{1}{k} \ln \left(\frac{2}{1+\mathrm{e}^{-2 k d(p, x)}}\right) .
$$

Recall that a point $x$ is a critical point of $d_{p}$ if for any vector $u \in T_{x} M$ there exists 
a minimal geodesic $\gamma$ from $x$ to $p$ so that $\angle\left(v, \gamma^{\prime}(0)\right) \leq \frac{\pi}{2}$.

From the inequality (28) and using the arguments of the proof of the Proposition 2.3 in [6], we deduce the following excess estimate for complete smooth metric measure space with $\operatorname{Ric}_{f} \geq 0$ and potential function bounded by $c \cdot d(p, x)$.

Theorem 3.10. Let $(M, g)$ be a complete noncompacte Riemannian manifold and $f$ a potential function on $M$ with $|f| \leq c d(p, x)$, for some fixed point $p, \quad$ Ric $_{f} \geq 0$ and $s(x)<\min \{d(p, x), d(q, x)\}$ then

$$
e_{p q}(x) \leq 8\left(\frac{s^{n}}{r}\right)^{1 / n-1} .
$$

By the same arguments as in [10] and using $\Delta_{h}$ instead of $\Delta$, one can prove the above lemma.

To prove the theorem 2.2, it suffices to show that $M$ contains no critical point of $d_{p}$ other than $p$.

For this, let $x$ be a point in M and $x \neq p$ and set $r=d(p, x)$. From the lemma 3.8 and the inequality (2) we have:

$$
d\left(x, \mathcal{R}_{p}\right)<\left(\frac{1}{8 k} \ln \left(\frac{2}{1+\mathrm{e}^{-2 k r}}\right)\right)^{\frac{n-1}{n}} \cdot r^{1 / n}
$$

hence, there exists a ray $\gamma$ issuing from $p$ verifying

$$
s=d(x, \gamma)<\left(\frac{1}{8 k} \ln \left(\frac{2}{1+\mathrm{e}^{-2 k r}}\right)\right)^{\frac{n-1}{n}} \cdot r^{1 / n}
$$

Let $q$ be a point on $\gamma$ so that $d(x, q)=d(x, \gamma)$ then $d(x, q)<r$. From the triangle inequality we have: $\min (d(p, x), d(\gamma, x))=s$ for all $t \geq 2 r$, which means $q \in \gamma([0,2 r])$. Such from the relations (44) and (45) we obtain

$$
e_{p, \gamma}(x) \leq e_{p, \gamma(2 r)}(x) \leq 8\left(\frac{s^{n}}{r}\right)^{1 /(n-1)}<\frac{1}{k} \ln \left(\frac{2}{1+\mathrm{e}^{-2 k r}}\right)
$$

The inequalities (43) and (47) show that $x$ is not a critical point of $d_{p}$. Hence, by isotopy lemma $M$ is diffeomorphic to $\mathbb{R}^{n}$.

\section{References}

[1] Wei, G. and Wylie, W. (2009) Comparison Geometry for the Bakry-Emery Ricci Tensor. Journal of Differential Geometry, 83, 377-405

[2] Limoncu, M. (2012) The Bakry-Émery Ricci Tensor and Its Applications to Some Compactness Theorems. Mathematische Zeitschrift, 271, 715-722. http://dx.doi.org/10.1007/s00209-011-0886-7

[3] Tadano, H. (2016) Remark on a Diameter Bound for Complete Riemannian Manifolds with Positive Bakry-Émery Ricci Curvature. Differential Geometry and Its Applications, 44, 136-143. http://dx.doi.org/10.1016/j.difgeo.2015.11.001

[4] Mahaman, B. (2001) Un théorème de Myers pour les variétés à courbure de Ricci minorée par une constante negative. Afrika Matematika, 12, 39-51. 
[5] Itokawa, Y. (1990) Distance Sphere and Myers-Type Theorems for Manifolds with Lower Bounds o, the Ricci Curvature. Illinois Journal of Matematics, 34, 693-705.

[6] Abresch, U. and Gromoll, D. (1990) On Complete Manifolds with Nonnegative Ricci Curvature. Journal of the American Mathematical Society, 3, 355-374.

http://dx.doi.org/10.1090/S0894-0347-1990-1030656-6

[7] Mahaman, B. (2000) A Volume Comparison Theorem and Number of Ends for Manifolds with Asymptotically Nonnegative Ricci Curvature. Revista Matemática Complutense, 13, 399-409.

[8] Mahaman, B. (2005) Open Manifolds with Asymptotically Nonnegative Curvature. Illinois Journal of Mathematics, 49, 705-717.

[9] Xia, C. (1999) Open Manifolds with Nonnegative Ricci Curvature and Large Volume Growth. Commentarii Mathematici Helvetici, 74, 456-466. http://dx.doi.org/10.1007/s000140050099

[10] Shen, Z. (1996) Complete Mnifolds with Nonnegative Ricci Curvature and Large Volume Growth. Inventiones Mathematicae, 125, 393-404.

http://dx.doi.org/10.1007/s002220050080

\section{Submit or recommend next manuscript to SCIRP and we will provide best service for you:}

Accepting pre-submission inquiries through Email, Facebook, LinkedIn, Twitter, etc. A wide selection of journals (inclusive of 9 subjects, more than 200 journals)

Providing 24-hour high-quality service

User-friendly online submission system

Fair and swift peer-review system

Efficient typesetting and proofreading procedure

Display of the result of downloads and visits, as well as the number of cited articles Maximum dissemination of your research work

Submit your manuscript at: http://papersubmission.scirp.org/

Or contact apm@scirp.org 